\title{
Validação de instrumento avaliativo da adesão às medidas de prevenção da tuberculose na atenção básica
}

\author{
Validation of an evaluation instrument of adherence to tuberculosis prevention measures in \\ primary care \\ Validación de un instrumento de evaluación de adhesión a medidas de prevención de tuberculosis \\ en atención primaria
}

\section{Resumo}

Objetivo: Desenvolver e validar um formulário para avaliação da adesão das unidades básicas de saúde às medidas de controle da tuberculose. Metodologia: Trata-se de um estudo metodológico para desenvolvimento de um questionário embasado nas literaturas nacionais e internacionais referente a temática. A validação de conteúdo e semântica aconteceu a partir do uso do Índice de Validade de Conteúdo (IVC) e o teste binominal, com a participação voluntária de 22 especialistas e 12 profissionais de saúde recrutados de 6 unidades básicas de saúde do distrito IV do município de Recife - PE incluídas no estudo. Resultado: O questionário obteve valores de IVC satisfatórios, em sua maioria maior ou igual a 0,80 , porém alguns itens não atingiram o valor mínimo do IVC, mas foram considerados significativos devido à aprovação pelo teste binominal, sendo maiores que a referência de 0,05 , com exceção de um item que foi retirado. Todas as sugestões dos juízes foram acatadas, a fim de tornar os itens mais coesos e compreensíveis. Conclusão: Dessa forma, o instrumento é considerado válido em conteúdo e semântica, contanto com três domínios: atividades administrativas, ações de controle ambiental e medidas de proteção individual, e 34 itens em sua versão final.

Palavras-chave: Tuberculose; Prevenção de doenças; Estudo de validação; Atenção primária à saúde.

\footnotetext{
Abstract

Objective: To develop and validate a form to assess the adherence of basic health units to tuberculosis control measures. Methodology: This is a methodological study to develop a questionnaire based on national and international literature on the subject. Content and semantic validation took place through the use of the Content Validity Index (CVI) and the binomial test, with the voluntary participation of 22 specialists and 12 health professionals recruited from 6 basic health units in district IV of the city of Recife - PE included in the study. Result: The questionnaire obtained satisfactory CVI values, mostly greater than or equal to 0.80 , but some items did not reach the minimum CVI value, but were considered significant due to approval by the binomial test, being greater than the
} 
reference of 0.05 , with the exception of one item that was withdrawn. All the judges' suggestions were accepted in order to make the items more cohesive and understandable. Conclusion: Thus, the instrument is considered valid in content and semantics, with three domains: administrative activities, environmental control actions and individual protection measures, and 34 items in its final version.

Keywords: Tuberculosis; Disease prevention; Validation study; Primary health care.

\section{Resumen}

Objetivo: Desarrollar y validar un formulario para evaluar la adherencia de las unidades básicas de salud a las medidas de control de la tuberculosis. Metodología: Se trata de un estudio metodológico para desarrollar un cuestionario basado en la literatura nacional e internacional sobre el tema. La validación de contenido y semántica se realizó mediante el uso del Índice de Validez de Contenido (CVI) y la prueba binomial, con la participación voluntaria de 22 especialistas y 12 profesionales de la salud reclutados de 6 unidades básicas de salud en el distrito IV de la ciudad de Recife - PE incluido en el estudio. Resultado: El cuestionario obtuvo valores de CVI satisfactorios, en su mayoría mayores o iguales a 0,80, pero algunos ítems no alcanzaron el valor mínimo de CVI, pero se consideraron significativos debido a la aprobación por la prueba binomial, siendo mayor que la referencia de 0.05 , con la excepción de un artículo que se retiró. Se aceptaron todas las sugerencias de los jueces para que los elementos fueran más coherentes y comprensibles. Conclusión: Así, el instrumento se considera válido en contenido y semántica, con tres dominios: actividades administrativas, acciones de control ambiental y medidas de protección individual, y 34 ítems en su versión final.

Palabras clave: Tuberculosis; Prevención de enfermedades; Estudio de validación; Primeros auxilios.

\section{Introdução}

A tuberculose constitui-se como uma doença infectocontagiosa transmitida de pessoa a pessoa através de gotículas de aerossóis. Está doença é causada por um microrganismo bacteriano denominado de Mycobacterium tuberculosis ou Bacilo de Koch (BK), afetando, na maioria dos casos, o pulmão, embora possa acometer outros órgãos (Mashabela, et al., 2019)

Atualmente, no contexto de Saúde Pública Mundial, a tuberculose é uma doença negligenciada e de caráter emergencial, principalmente, em países em desenvolvimento como o Brasil. Em 2019, estimou-se 10 milhões de novos casos de tuberculose em todo o mundo, e 1,4 milhão de 9 mortes decorrentes da doença (OMS, 2020). Ainda, em 2020, o Brasil notificou 66.819 casos novos de TB, e um coeficiente de incidência de 31,6 casos por 100 mil habitantes (Brasil, 2020)

No Brasil, apesar de ser considerada uma doença com diagnóstico e tratamento realizados de forma universal e gratuita pelo Sistema Único de Saúde (SUS), apresentam-se, ainda, barreiras no acesso e a não adesão integral aos protocolos de controle nos serviços de saúde, seja por falta de recursos ou seu gerenciamento, repercutindo em fornecimentos de medidas e serviços prestados incoerentes às recomendações mundiais (Brasil, 2017), (Sousa, et al.,2020).

Emerge o papel da atenção primária como ponto estrutural para o desenvolvimento de ações que favoreçam medidas de prevenção da tuberculose, através da aplicação efetiva de protocolos investigativos para o controle doença, de forma a garantir a promoção, proteção e continuidade de cuidados integrais e eficazes à comunidade, garantindo, assim, o direito civil à saúde (Brasil, 2002).

Diante disso, demonstra-se essencial a avaliação contínua do processo de trabalho na atenção primária, bem o desenvolvimento de instrumentos avaliativos organizacionais em saúde, considerando as peculiaridades dos serviços relacionados à tuberculose, através de uma assistência multidisciplinar, atribuída a partir de conhecimentos técnico-científicos, garantindo a integralidade em saúde. A aplicabilidade de novas tecnologias em saúde, na atenção primária, promove impactos positivos, referentes à acessibilidade, qualidade assistencial, e prevenção em saúde, e, especialmente, reduzir as lacunas no acesso a informações (Kanchar, A \& Swaminathan, S, 2019).

Ao reconhecer as especificidades deste contexto em saúde, o presente estudo objetivou desenvolver e validar, um formulário para avaliação da adesão das unidades básicas de saúde às medidas de controle de infecção da tuberculose, podendo com isso estruturar a primeira etapa para a construção de indicadores relevantes para avaliação do desempenho e continuação 
dos modelos assistenciais instituídos. Quais são as evidências de validade de conteúdo do formulário para avaliação da adesão das unidades básicas de saúde às medidas de prevenção e controle da Tuberculose?

\section{Metodologia}

Estudo metodológico, o qual delineia-se a partir da investigação, organização e análise de dados, objetivando, a partir disso, construir, validar e avaliar instrumentos e formas de pesquisa, com a finalidade de melhorar a confiabilidade e validade dessas ferramentas (Vieira, M. A, et al., 2016).

A construção e validação do questionário foi planejado de acordo com o modelo sugerido por Pasquali (2010), adaptado à pesquisa, se baseando em cinco etapas: Teórica; Observação em campo; Construção dos itens; Validação de conteúdo; Validação semântica junto ao público alvo. Contemplando a fase teórica, inicialmente, foi levantado estudos bibliográficos acerca da temática para desenvolvimento dos itens, conceitos e estudos sobre a tuberculose; medidas de capacitação à atenção básica no que tange ao controle e prevenção contra a tuberculose; e estudos sobre validação de questionários em saúde.

No intuito de contemplar informações relevantes às necessidades do presente instrumento, a segunda etapa do estudo foi realizada a partir de observação em campo, de forma virtual, no município de Recife - PE, local de aplicabilidade de validação semântica do estudo. Está etapa foi viável por meio dos sistemas de informação em saúde fornecido pelo SUS, bem como sua plataforma em saúde o DATASUS, através do detalhamento de dados de morbidade, mortalidade e índices referentes a tuberculose na localidade alvo, a fim de se obter um parâmetro acerca da temática na região.

Posteriormente, iniciou-se a terceira parte do estudo, englobando o desenvolvimento do questionário, inicialmente, composto por 35 questões fechadas, com os itens organizados dicotomicamente, com ênfase no Manual de Recomendações para o Controle da Tuberculose. O instrumento dividiu-se em três domínios: Medidas administrativas, Medidas ambientais e Medidas de proteção individual.

A quarta etapa do estudo contemplou o processo de validação de conteúdo do instrumento avaliativo organizacional em saúde, a qual contou com a participação de 23 juízes especialistas na temática abordada, seguindo critérios para construção do painel de juízes, propostos por Fehring(1994), incluindo-se aqueles que apresentaram conhecimentos e produções científicas recentes a temáticas (Infectologia; saúde pública e/ou tecnologia em saúde ou em gestão em saúde e/ saúde coletiva) e excluídos os que apresentaram produções científicas que não se relacionavam à temática e ao público-alvo da pesquisa em questão. A seleção dos Juízes ocorreu por levantamento na plataforma Lattes.

O desenvolvimento metodológico da quinta etapa do estudo ocorreu mediante a validação semântica do questionário junto ao público alvo, tendo como cenário 6 Unidades Básicas de Saúde do Distrito IV do município de Recife no estado de Pernambuco. O estudo contou com a participação de 12 profissionais de saúde, para avaliação semântica, adotando-se como critérios de inclusão profissional ser enfermeiro e/ou médico e/ou técnico de enfermagem que atue no tratamento de tuberculose na região correspondente ao estudo, excluindo-se aqueles que apresentaram limitações ou alterações de aspecto físico, emocional ou cognitivo que impliquem sua participação ou profissionais que estão afastados por motivos médicos ou administrativos como férias.

Para análise da validação do instrumento foi construído um banco de dados no programa EPI INFO. O banco foi exportado para o software PSPP. Em associação, para caracterizar o perfil pessoal e profissional dos juízes avaliados foram calculadas as frequências percentuais e construídas as distribuições de frequência.

Ainda, foi obtida a caracterização da experiência profissional dos juízes assim como da produção científica. A validade do conteúdo do instrumento proposto foi avaliada pelo IVC e aplicado o teste Binomial para verificar a significância 
da adequação em comparação ao valor mínimo determinado pela literatura $(0,80)$. Todas as conclusões apresentadas foram consideradas no nível de significância de 5\%.

A pesquisa foi desenvolvida seguindo apreciação e aprovação pelo Comitê de Ética em Pesquisa, do Centro de Ciências da Saúde, da Universidade Federal de Pernambuco. Seguindo o que dispõe a Resolução 466/12 que envolve pesquisa com seres humanos, do Conselho Nacional de Saúde do Ministério da Saúde, considerando os aspectos da autonomia, não maleficência, beneficência e justiça, sob número do parecer: 4.472.769.

\section{Resultados}

A etapa de observação em campo, foi realizada para o levantamento dos dados referente ao parâmetro situacional da tuberculose contendo os indicadores de incidência da morbidade desta doença na população do Município de Recife, por meio das ferramentas do Ministério da Saúde, desenvolvidos pelo Departamento de informações do Sistema Único de Saúde DATASUS. Além disso, utilizou-se também o Sistema de Sala de Apoio à Gestão (SAGE), para analisar a situação de saúde sobre a tuberculose, obtendo-se, assim, dados referentes a taxas e percentuais em todo o município.

O município de Recife notificou, no ano de 2020, 2.360 casos de Tuberculose, ainda, considerando o tipo de entrada, foram notificados 1.686 casos novos, 147 casos recidivas; 336 de casos de reingresso após abandono; 89 casos de transferências; 86 confirmados apenas após óbito e 16 considerados "não sabe".

Ademais, calculou-se o coeficiente de incidência por meio do número de casos novos por 100 mil habitantes na população residente e a última estimativa populacional de acordo com o Instituto Brasileiro de Geografia e Estatística, de 1.653.461 pessoas no ano de 2020 no município de Recife. Obtendo assim, um coeficiente de incidência de tuberculose de 102 casos a cada 100.000 habitantes nesse ano (IBGE, 2020), (Brasil, 2020).

Posteriormente, iniciou a construção do questionário, que foi desenvolvido a partir de três domínios que abarcam as principais diretrizes de prevenção e controle para tuberculose, instaurados no Manual de Recomendações para o Controle da Tuberculose, bem como contemplando ações de maior relevância no parâmetro preventivo da tuberculose nos serviços de saúde em nível nacional e internacional.

A primeira versão do questionário foi composta por 35 questões fechadas, com os itens organizados dicotomicamente intitulados em três domínios: medidas administrativas, medidas ambientais e medidas de proteção individual.

O domínio das medidas administrativas abarcou o desenvolvimento e implementação de ações e protocolos que assegurem a prevenção e o controle da tuberculose nas unidades e na vida da comunidade. Em continuidade, o domínio das Medidas Ambientais objetivou a instituição de itens que abrangem a minimização da disseminação de gotículas e aerossóis infectantes no ar. Articulou-se a implementação de meios favoráveis à prevenção de contaminação pela ventilação local.

Por conseguinte, o domínio de Medidas Ambientais incorpora ações e equipamentos de proteção individual, que são de suma importância na prevenção de possíveis infecções pelos profissionais de saúde, bem como no controle da propagação de conteúdos infectantes no ar por usuários diagnosticados com tuberculose ativa ou suspeitos respiratórios.

A validação do conteúdo do instrumento contou com uma distribuição de perfil pessoal e profissional dos juízes da pesquisa, em sua maioria com idade de mais de 50 anos (47,8\%), formados em enfermagem (100,0\%), possuindo título de doutor $(69,6 \%)$, em sua maioria mulheres $(82,6 \%)$ e pernambucanos $(82,6 \%)$.

Os especialistas apreciaram o conteúdo do questionário desenvolvido, de forma a determinar se os domínios estavam adequados ou inadequados, em relação a abrangência de cada item incluído, assim como a relevância dos conceitos envolvidos, como também a clareza quanto sua redação, verificando se foram redigidos de forma compreensível para atingir os objetivos propostos. 
Na Tabela 1 temos a avaliação do grau de concordância dos juízes participantes acerca da adequação dos domínios do estudo. Verifica-se que os domínios 1,2 e 3 apresentaram I-CVI menor que 0,80, para expressividade do conteúdo, porém o teste binominal não apresentou significância em relação ao valor de referência. Para a permanência dos domínios foi apresentado I-CVI maior ou igual a 0,80 em todos os domínios, indicando alto nível da relevância dos domínios.

Tabela 1. Concordância dos participantes acerca da adequação Domínios avaliados.

\begin{tabular}{|c|c|c|c|c|}
\hline $\begin{array}{c}\text { Domínio/questão } \\
\text { avaliada }\end{array}$ & $\mathbf{N}$ & $\%$ & I-CVI ${ }^{1}$ & p-valor \\
\hline $\begin{array}{l}\text { O Domínio } 1 \text { realmente } \\
\text { expressa seu conteúdo? }\end{array}$ & 17 & 73,9 & 0,74 & $0,305^{2}$ \\
\hline $\begin{array}{l}\text { Os itens do Domínio } 1 \text { devem } \\
\text { permanecer nesse domínio? }\end{array}$ & 23 & 100 & 1,0 & $0,006^{3}$ \\
\hline $\begin{array}{l}\text { O Domínio } 2 \text { realmente } \\
\text { expressa seu conteúdo? }\end{array}$ & 18 & 78,3 & 0,78 & $0,499^{2}$ \\
\hline $\begin{array}{l}\text { Os itens do Domínio } 2 \text { devem } \\
\text { permanecer nesse domínio? }\end{array}$ & 23 & 100 & 1,0 & $0,006^{3}$ \\
\hline $\begin{array}{l}\text { O Domínio } 3 \text { realmente } \\
\text { expressa seu conteúdo? }\end{array}$ & 16 & 69,6 & 0,70 & $0,160^{2}$ \\
\hline $\begin{array}{l}\text { Os itens do Domínio } 3 \text { devem } \\
\text { permanecer nesse domínio? }\end{array}$ & 20 & 87,0 & 0,87 & $0,297^{3}$ \\
\hline
\end{tabular}

1Índice de validade de conteúdo. ${ }^{2} \mathrm{p}$-valor do teste Binomial $\left(\mathrm{H}_{0}: \mathrm{p}=0,80 \times \mathrm{H}_{1}: \mathrm{p}<0,80\right) .{ }^{3} \mathrm{p}$ valor do teste Binomial $\left(\mathrm{H}_{0}: \mathrm{p}=0,80 \times \mathrm{H}_{1}: \mathrm{p}>0,80\right)$. Fonte: Autores.

Na Tabela 2, todos os itens do domínio 2 apresentaram o I-CVI maior que 0,80 para a clareza, exceto para os itens 4, 10, 17 e 18. Apesar de apresentarem I-CVI menor que a referência, o teste binominal não foi significativo, indicando que os itens satisfazem o critério mínimo para aprovação. Para representatividade, todos os itens apresentaram I-CVI maior de 0,80 e todos apresentaram significância estatística no teste binominal. 
Tabela 2. Concordância dos participantes acerca da adequação dos itens avaliados.

\begin{tabular}{|c|c|c|c|c|c|c|c|c|}
\hline \multirow{2}{*}{ Domínio/questão avaliada } & \multicolumn{4}{|c|}{ CLAREZA } & \multicolumn{4}{|c|}{ REPRESENTATIVIDADE } \\
\hline & $\mathbf{N}$ & $\%$ & I-CVI' & p-valor & $\mathbf{N}$ & $\%$ & I-CVI' & p-valor \\
\hline $\begin{array}{l}\text { 1.1 Existe um protocolo ou } \\
\text { planejamento de ação } \\
\text { para uma rápida identificação } \\
\text { e condução do sintomático } \\
\text { respiratório? }\end{array}$ & 20 & 87 & 0,87 & $0,297^{3}$ & 22 & 95,7 & 0,96 & $0,040^{3}$ \\
\hline $\begin{array}{l}\text { 1.2 Utiliza-se o protocolo de } \\
\text { duração de tosse de no } \\
\text { mínimo duas semanas ou } \\
\text { mais para rastreio de SR? }\end{array}$ & 22 & 95,7 & 0,96 & $0,040^{3}$ & 22 & 95,7 & 0,96 & $0,040^{3}$ \\
\hline $\begin{array}{l}1.3 \text { A equipe de atenção } \\
\text { básica possui registro do } \\
\text { número de usuários de } \\
\text { tuberculose? }\end{array}$ & 20 & 87 & 0,87 & $0,297^{3}$ & 23 & 100 & 1,00 & $0,006^{3}$ \\
\hline $\begin{array}{l}1.4 \text { A equipe realiza a } \\
\text { notificação de casos de } \\
\text { tuberculose na unidade? }\end{array}$ & 18 & 78,3 & 0,78 & $0,499^{2}$ & 22 & 95,7 & 0,96 & $0,040^{3}$ \\
\hline $\begin{array}{l}1.5 \text { Quando notificado um } \\
\text { caso de SR, é oferecido } \\
\text { imediatamente a máscara } \\
\text { cirúrgica para uso durante sua } \\
\text { permanência na unidade? }\end{array}$ & 20 & 87 & 0,87 & $0,297^{3}$ & 23 & 100 & 1,00 & $0,006^{3}$ \\
\hline $\begin{array}{l}\text { 1.6 Quando notificado um } \\
\text { caso de SR, instrui-se o } \\
\text { mesmo com relação à } \\
\text { etiqueta da tosse (levar o } \\
\text { braço ou lenço à boca quando } \\
\text { tossir)? }\end{array}$ & 20 & 87 & 0,87 & $0,297^{3}$ & 22 & 97,5 & 0,96 & $0,040^{3}$ \\
\hline $\begin{array}{l}1.7 \text { A unidade realiza o } \\
\text { protocolo de controle de } \\
\text { contatos referentes a TB? }\end{array}$ & 21 & 91,3 & 0,91 & $0,133^{3}$ & 23 & 100 & 1,00 & $0,006^{3}$ \\
\hline $\begin{array}{l}1.8 \text { Nesta unidade, há a } \\
\text { realização de baciloscopia } \\
\text { direta de escarro? }\end{array}$ & 21 & 91,3 & 0,91 & $0,133^{3}$ & 22 & 95,7 & 0,96 & $0,040^{3}$ \\
\hline
\end{tabular}


1.9 Nesta unidade, há a realização Rápido Molecular para TB (TM-TB)?

1.10 Nesta unidade é fornecido, quando necessário, a vacinação de BCG?

$1.11 \mathrm{~A} 1^{\mathrm{a}}$ amostra de escarro para o diagnóstico de tuberculose é coletada na primeira abordagem/consulta com o usuário?

1.12 A dispensação de medicamentos para o tratamento da TB é realizada na unidade?

1.13 Nesta unidade, é estabelecido um fluxo de atendimento baseado nos casos positivos e negativos à baciloscopia ou TRM-TB?

1.14 Pessoas SR e/ou infectadas ainda, com Tuberculose pulmonar ou laríngea esperam por atendimento ou

procedimentos em áreas mais ventiladas?

1.15 Pessoas SR e/ou infectadas ainda, com Tuberculose pulmonar ou laríngea são consideradas prioritárias na procura por atendimento na unidade, independente do motivo da procura?

1.16 Nesta unidade, há a realização do Tratamento Diretamente Observado (TDO)?
20

87

0,87

$0,297^{3}$

23

100

1,00

$0,006^{3}$

18

78,3

0,78

$0,499^{2}$

23

100

1,00

$0,006^{3}$

22

95,7

0,96

$0,040^{3}$

23

100

1,00

$0,006^{3}$

$21 \quad 91,3$

$0,133^{3}$

23

100

1,00

$0,006^{3}$

$\begin{array}{llllllll}23 & 100 & 1,00 & 0,006^{3} & 23 & 100 & 1,00 & 0,006^{3}\end{array}$

$\begin{array}{llllllll}19 & 82,6 & 0,83 & 0,501^{3} & 22 & 95,7 & 0,96 & 0,040^{3}\end{array}$

u

$\begin{array}{llllllll}9 & 82,6 & 0,83 & 0,501^{3} & 23 & 100 & 1,00 & 0,006^{3}\end{array}$

$\begin{array}{llllllll}23 & 100 & 1,00 & 0,006^{3} & 23 & 100 & 1,00 & 0,006^{3}\end{array}$


Research, Society and Development, v. 11, n. 1, e1011124148, 2022

(CC BY 4.0) | ISSN 2525-3409 | DOI: http://dx.doi.org/10.33448/rsd-v11i1.24148

1.17 A unidade fornece

Projeto Terapêutico Singular

$17 \quad 73,9$

0,74

$0,305^{2}$

22

95,7

0,96

$0,040^{3}$

(PTS)?

1.18 A unidade de saúde

fornece Grupos de adesão

e/ou atividades lúdicas e/ou

oferta de incentivos

direcionados à tuberculose?

1.19 A equipe realiza busca

ativa para Faltosos do

tratamento (até o período de

20

87

0,87

$0,297^{3}$

23

100

1,00

$0,006^{3}$

29 dias)?

1.20 A equipe realiza busca

ativa para abandono do

tratamento (superior a 30

$21-91,3$

0,91

$0,133^{3}$

23

100

1,00

$0,006^{3}$

dias)?

1.21 Nesta unidade há a promoção de programas ou

cursos de educação

20

87

0,87

$0,297^{3}$

23

100

1,00

$0,006^{3}$

permanente referentes ou

associados a Tuberculose?

${ }^{1}$ Índice de validade de conteúdo. ${ }^{2} \mathrm{p}$-valor do teste Binomial $\left(\mathrm{H}_{0}: \mathrm{p}=0,80 \times \mathrm{H}_{1}: \mathrm{p}<0,80\right) .{ }^{3} \mathrm{p}$-valor do teste Binomial $\left(\mathrm{H}_{0}: \mathrm{p}=0,80 \times \mathrm{H}_{1}: \mathrm{p}>0,80\right)$. Fonte: Autores.

Na Tabela 3, todos os itens do domínio 2 apresentaram I-CVI maior de 0,80 e todos apresentaram significância estatística no teste binominal quanto à sua clareza e representatividade. 
Tabela 3. Concordância dos participantes acerca da adequação dos itens avaliados.

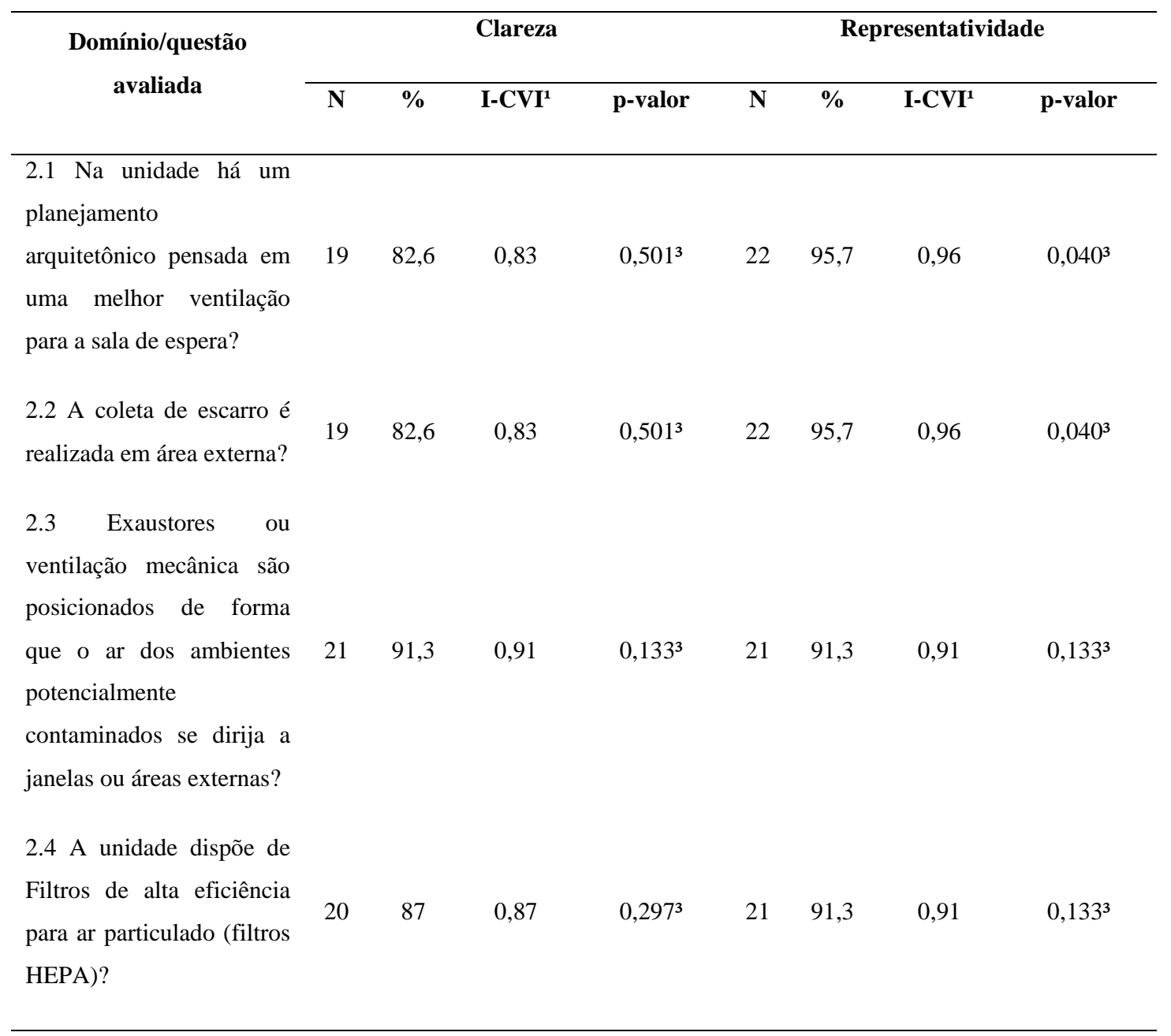

1Índice de validade de conteúdo. ${ }^{2} \mathrm{p}$-valor do teste Binomial $\left(\mathrm{H}_{0}: \mathrm{p}=0,80 \times \mathrm{H}_{1}: \mathrm{p}<0,80\right) .{ }^{3} \mathrm{p}$-valor do teste Binomial $\left(\mathrm{H}_{0}: \mathrm{p}=0,80 \times \mathrm{H}_{1}: \mathrm{p}>0,80\right)$. Fonte: Autores.

Na Tabela 4, todos os itens do domínio 3 apresentaram o I-CVI maior que 0,80 para a clareza, exceto para os itens 2, 6 e 10. Os itens 6 e 10 mesmo apresentando I-CVI menor que a referência do teste binominal não foi significativo, indicando que os itens satisfazem o critério mínimo para aprovação. Porém o item 2 apresentou o teste binominal significativo necessitando de sua correção quanto a sua clareza. Para representatividade, todos os itens apresentaram I-CVI maior de 0,80, com exceção ao item 2, apesar disso seu teste binominal não foi significativo e com isso demonstrando a satisfação do item ao critério mínimo de aprovação. 
Tabela 4. Concordância dos participantes acerca da adequação dos itens avaliados.

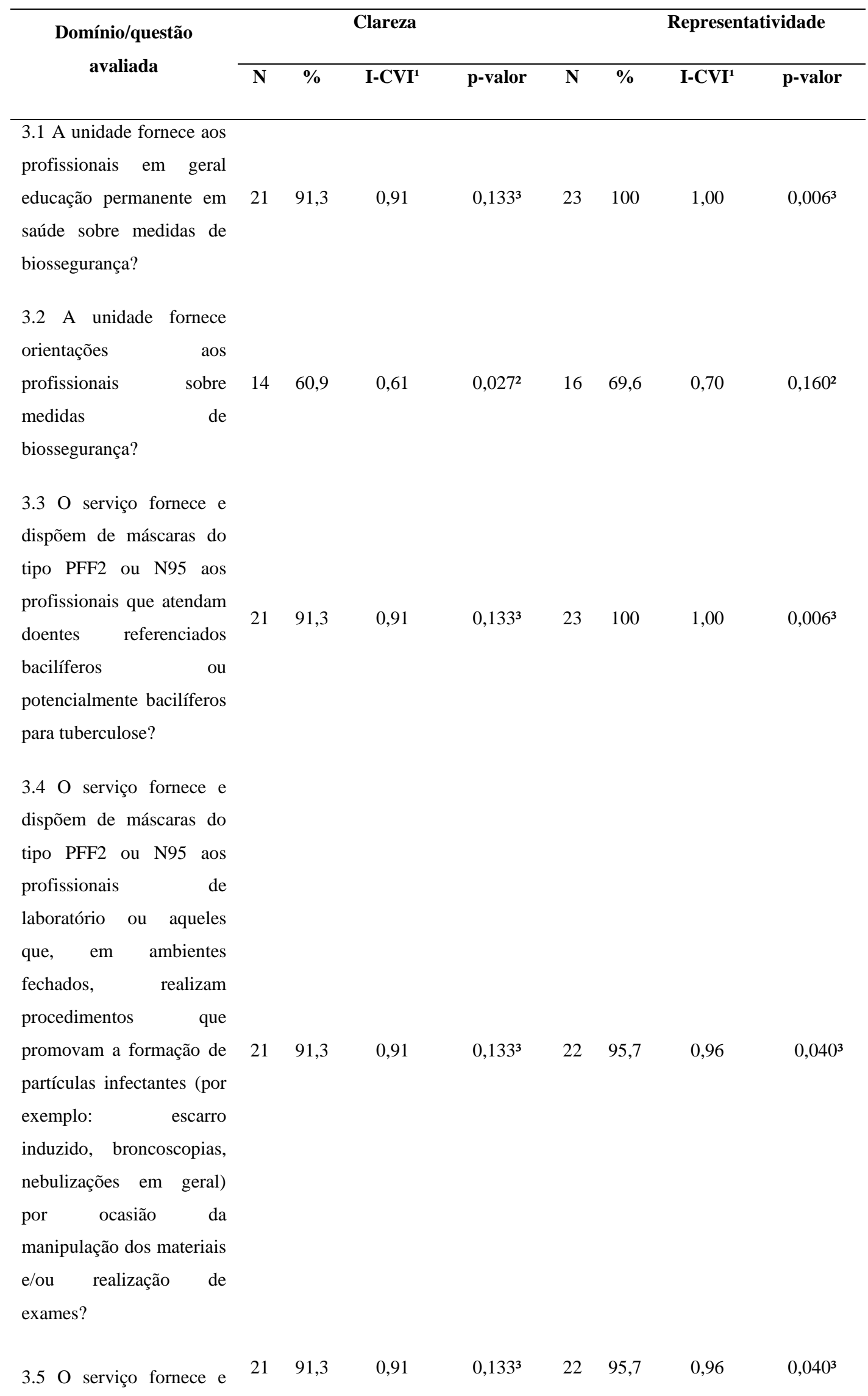




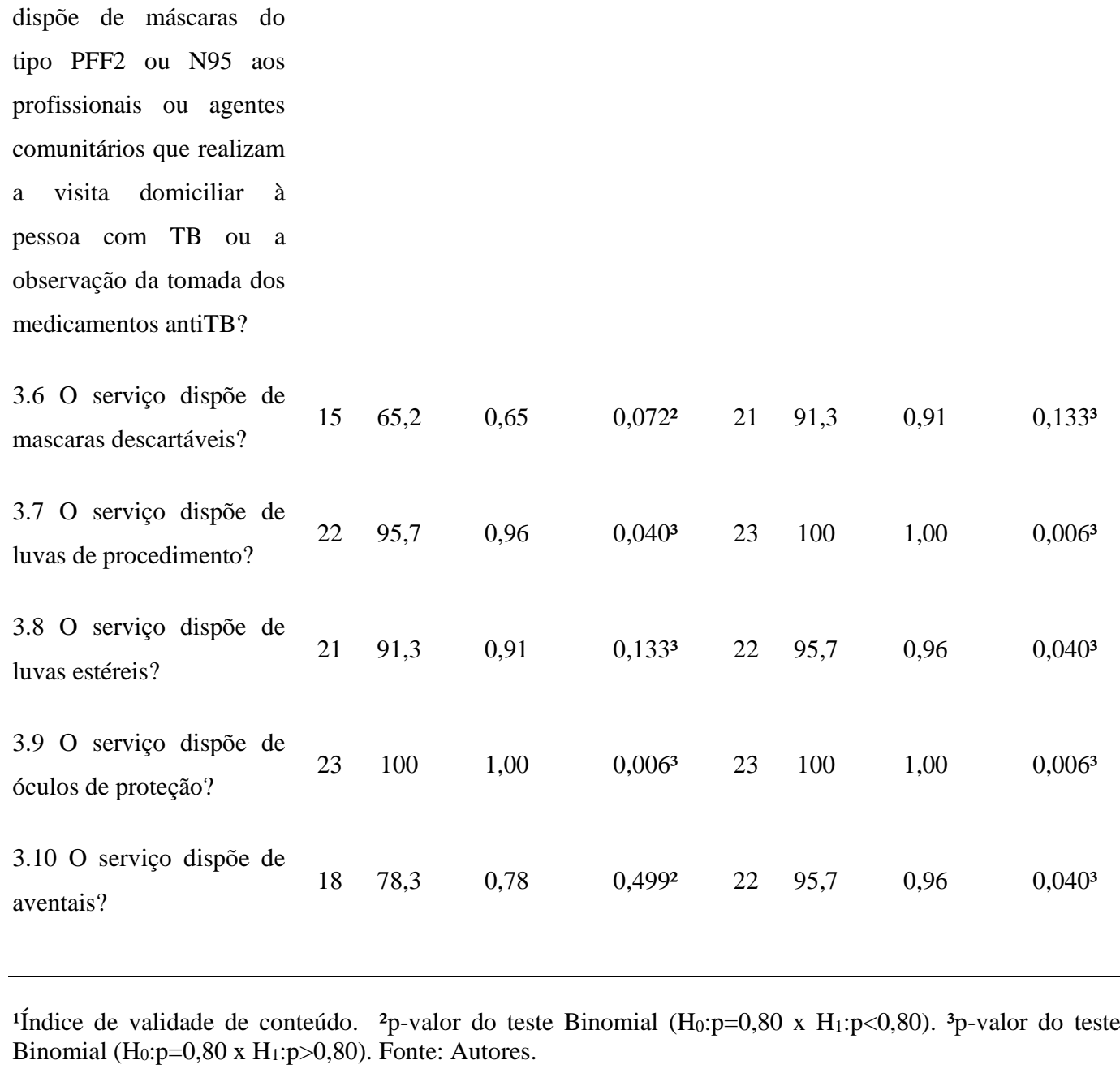

1Índice de validade de conteúdo. ${ }^{2} \mathrm{p}$-valor do teste Binomial $\left(\mathrm{H}_{0}: \mathrm{p}=0,80 \times \mathrm{H}_{1}: \mathrm{p}<0,80\right) .{ }^{3} \mathrm{p}$-valor do teste Binomial $\left(\mathrm{H}_{0}: \mathrm{p}=0,80 \times \mathrm{H}_{1}: \mathrm{p}>0,80\right)$. Fonte: Autores.

Em todos os itens avaliados, foram consideradas as mudanças solicitadas e sugeridas pelos juízes especialistas, realizando as modificações necessárias, mesmo diante da significância atingida. Todos os juízes especialistas apresentaram algum tipo de comentário ou sugestão para o aprimoramento e coesão do questionário. Ademais, os especialistas destacaram, nos comentários, a relevância da construção de um instrumento que viabiliza a avaliação acerca do controle e a prevenção da tuberculose na atenção básica, através da perspectiva de uma gestão e assistência profissional eficiente e apta aos protocolos e manuais formulados pelo Ministério da saúde.

Nesse sentido, levando-se em consideração a avaliação final dos especialistas, foram alterados os títulos dos domínios 1 e 2, passando a ser Atividades administrativas e Ações de controle ambiental, respectivamente, como também houve alteração em alguns termos, objetivando a compreensão do público alvo. No entanto, assentindo as sugestões dos especialistas, bem como a significância estatística de um dos itens quanto a sua correção, foi realizada sua retirada do instrumento avaliativo. Diante disso, a versão final do questionário resultou em 34 questões satisfatoriamente validadas.

Em seguimento, foi realizada a validação semântica junto ao público alvo, com a participação de 12 profissionais de saúde que trabalham nas 6 unidades básicas de saúde incluídas no estudo, dentre eles, participaram enfermeiros e técnicos de enfermagem.

$\mathrm{Na}$ Tabela 5, foi demonstrada a avaliação do grau de concordância dos profissionais de saúde participantes acerca da semântica do estudo. Verificou-se que o item 2.1 no enquadramento de organização apresentou I-CVI menor que 0,80, para expressividade do conteúdo, porém o teste binominal não apresentou significância em relação ao valor de referência. Em 
relação aos outros itens, apresentaram I-CVI maior ou igual a 0,80 em todos os aspectos, indicando alto nível da relevância dos itens.

Tabela 5. Concordância dos profissionais de saúde sobre a semântica do questionário.

\begin{tabular}{llllll}
\hline Aspecto abordado & N & $\%$ & I-CVI $^{1}$ & p-valor \\
\hline 1 & OBJETIVOS & & & & \\
\hline
\end{tabular}

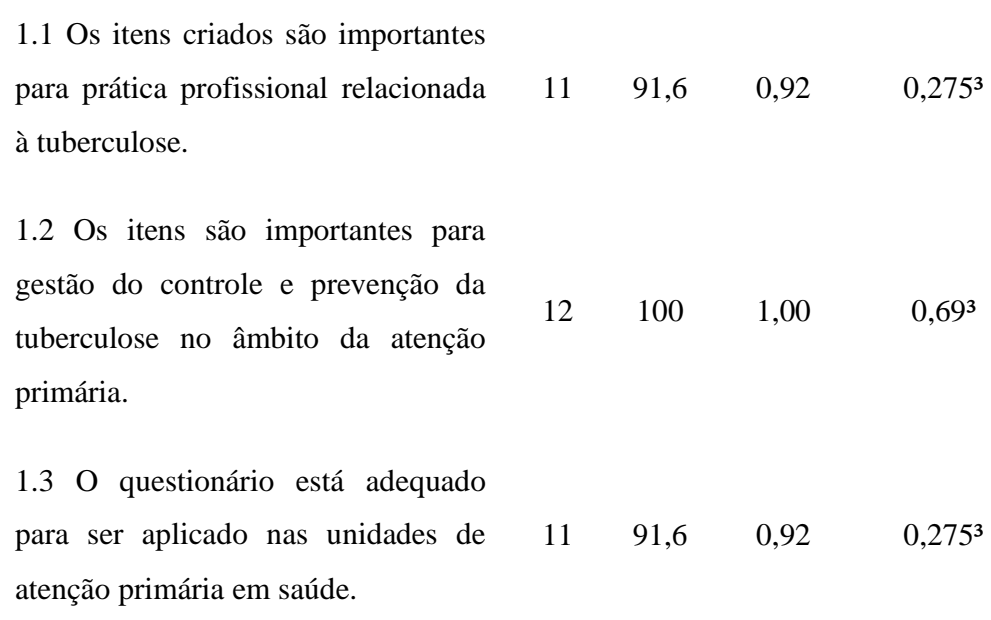

\section{ORGANIZAÇÃO}

\begin{tabular}{|c|c|c|c|c|}
\hline $\begin{array}{l}2.1 \text { Há coerência entre os domínios e } \\
\text { os itens. }\end{array}$ & 9 & 75 & 0,75 & $0,442^{2}$ \\
\hline $\begin{array}{l}2.2 \mathrm{O} \text { título é atraente e indica o } \\
\text { conteúdo do questionário. }\end{array}$ & 10 & 83 & 0,83 & $0,558^{3}$ \\
\hline
\end{tabular}

\section{ESTILO DA ESCRITA}

3.1 A escrita está clara e objetiva. $\quad 11 \quad 91,6 \quad 0,92 \quad 0,275^{3}$

3.2 O vocabulário corresponde ao nível de conhecimento da equipe de $\begin{array}{lllll}11 & 91,6 & 0,92 & 0,275^{3}\end{array}$ saúde.

\section{APARÊNCIA}
4.1 As seções parecem organizadas
11
91,6
0,92
$0,275^{3}$

1Índice de validade de conteúdo. ${ }^{2} \mathrm{p}$-valor do teste Binomial $\left(\mathrm{H}_{0}: \mathrm{p}=0,80 \mathrm{x}\right.$ $\left.\mathrm{H}_{1}: \mathrm{p}<0,80\right)$. ${ }^{3} \mathrm{p}$-valor do teste Binomial $\left(\mathrm{H}_{0}: \mathrm{p}=0,80 \times \mathrm{H}_{1}: \mathrm{p}>0,80\right)$. Fonte: Autores. 


\section{Discussão}

O desenvolvimento do presente estudo possibilitou a construção e validação de um questionário, para ser aplicado nas unidades básicas de saúde, com o objetivo de avaliar a adesão das unidades básicas de saúde às medidas de controle e prevenção da tuberculose. Em continuidade foi apresentada a perspectiva do papel primordial da Atenção Primária em Saúde como porta de entrada no Sistema Único de Saúde, ao destacar-se como um local favorável para a resolubilidade assistencial; a comunicação com os fluxos de pessoas, informações e produtos; e a responsabilidade da organização dos micro territórios sanitários, no desencadeamento de atributos para prevenção e controle da tuberculose (Pelissari, 2018).

A etapa de observação em campo favoreceu a busca de referencial teórico a partir de dados secundários informatizados em saúde, repercutindo na valorização de tecnologias que processam e disseminam informações acerca da saúde populacional, tais como o Departamento de informações do Sistema Único de Saúde (D'Onofrio, B. M, et al.,2020).

Para considerar a qualidade e confiabilidade do instrumento organizacional avaliativo, o estudo contou com a validação de conteúdo com juízes especialistas na temática alvo, para fundamentar um consenso acerca da representatividade e clareza dos itens (Azeredo, et al., 2019). A validação de conteúdo de instrumentos em saúde proporciona uma análise metodológica que expressa a aplicabilidade mensurável de um respectivo fenômeno (Tolentino,2019).

Compondo a validação, os juízes selecionados precisavam ter expertise comprovada na área, seguindo o sistema de pontuação de Fehring ${ }^{9}$ (1994) mínima de 5 pontos. Os dados obtidos na caracterização do perfil profissional e acadêmico dos juízes corroboram com a informação supracitada ao demonstrar que o equivalente a $70 \%$ possui como maior titulação doutorado, TCC e/ou dissertação e/ou tese envolvendo estudos dentre a temática abordada na pesquisa. Os níveis de titulação influem no acréscimo do conhecimento científico dos juízes, favorecendo na criticidade e julgamentos mais robustos e com isso, resultados mais verídicos (Macedo \& Bohomol, 2019)

O tempo de experiência prática dos especialistas na área estudada, também foi considerado um fator de grande importância e está diretamente relacionado com a capacidade de inferências dos juízes. Foram quantificados no estudo que 91\% dos especialistas participantes que abarcavam uma mínima de prática profissional na temática de 3 anos, logo, considerou-se que o tempo referido está dentro das recomendações dos estudiosos sobre validação de conteúdo. Para Simmons et al (2003), o estabelecimento de um tempo mínimo é de dois anos de prática profissional.

Em relação à concordância dos juízes quanto à representatividade e clareza dos itens e domínios, o questionário obteve valores de IVC satisfatórios, em sua maioria maior ou igual a 0,80 , em contemplação com as orientações advindas da literatura. Porém alguns itens não atingiram o valor mínimo do IVC, mas foram considerados significativos devido à aprovação pelo teste binominal, sendo maiores que a referência de 0,05 , com exceção de um item que foi retirado. Todas as sugestões dos juízes foram acatadas, a fim de tornar os itens mais coesos, claros e compreensíveis. Cumpre destacar que a seleção do conteúdo abrangido pelo questionário está de acordo com as competências e deveres a serem desenvolvidos pelas unidades básicas de saúde.

Em destreza a clareza, visualização e compreensão na leitura dos itens pelo público alvo da aplicabilidade do instrumento, realizou-se a validação semântica, no sentido de englobar a necessidade dos aspectos mencionados, tendo em vista uma avaliação direta com o grupo alvo a ser instaurado o questionário. A aplicabilidade e análise de aceitação prévia do instrumento junto a este público, favorece ao pesquisador identificar as demandas específicas que permeiam o cenário de estudo, bem como o rigor científico positivo no processo de tornar o estudo válido e aplicável (Wisdom, 2012).

O questionário foi considerado validado semanticamente, dado que os valores do IVC foram superiores a $80 \%$, com exceção de um item referente à organização, embora que apresente IVC menor que o referenciado, o teste binominal não foi significativo, satisfazendo o critério mínimo para aceitação. 
Nessa perspectiva, o questionário desenvolvido demonstrou ser uma ferramenta que viabiliza a avaliação direta com as unidades de atenção primária em saúde, e a validação mostrou a importância de se escolher cuidadosamente as palavras que permitam o entendimento compatível com o público alvo e seu aspecto profissional. Essa apresentação, é relevante considerando-se que, na área da saúde, frequentemente divulgam-se as informações que podem dificultar o entendimento pelo público-alvo (Ribeiro, 2020).

\section{Conclusão}

O presente instrumento de estudo foi considerado válido em conteúdo e semântica pelos especialistas e profissionais de saúde. Sendo benéfico à comunidade científica e a sociedade, por englobar produções de dados regionais atualizados, trazendo para a temática maior atenção e credibilidade, bem como na perspectiva de estimular a produção de novos trabalhos que abarquem o tema em questão, com isso, novas ações de melhoria e qualidade, instigando a implementação de ferramentas que auxiliem o manejo do cuidado em todos os níveis de integralidade em saúde relacionados à Tuberculose.

\section{Referências}

Azeredo, L. G., Diaz, C. M. G., Souza, M. H. T. de Costenaro, R. G. S., \& Portela, J. de L. (2019). Construction and validation of educational technology about Congenital Syphilis. Research, Society and Development, 8(12), e458121939. https://doi.org/10.33448/rsd-v8i12.1939.

Brasil (2020). Departamento de Vigilância das Doenças Transmissíveis, Secretaria de Vigilância em Saúde, Ministério da Saúde. Boletim Epidemiológico Tuberculose.Brasília: Ministério da Saúde.

Brasil (2019). Departamento de Vigilância Epidemiológica. Secretaria de Vigilância em Saúde, Ministério da Saúde. Manual de recomendações para o controle da tuberculose no Brasil. Brasília: Ministério da Saúde.

Brasil (2017). Secretaria de Vigilância em Saúde, Ministério da Saúde. Plano nacional pelo fim da tuberculose. Brasília: Ministério da Saúde.

Brasil (2017). Departamento de Atenção Básica. Secretaria de Políticas à Saúde, Ministério da Saúde. Caderno de Atenção Básica: Manual Técnico para o Controle da Tuberculose. Brasília: Ministério da Saúde.

D'Onofrio, B M; Sjölander, A; Lahey, B B; Lichtenstein, P \& Öberg, A S. (2020). Accounting for Confounding in Observational Studies. Annual review of clinical psychology, 16, 25-48. https://doi.org/10.1146/annurev-clinpsy-032816-045030

Fehring R (1994). The Fehring Model. In Carrol-Johnson R, Paquete M, editores. Classification of nursing diagnoses: proceedings of the tenth conference of North American Nursing Diagnosis Association. Philadelphia: Lippincott, p. 55-62.

IBGE - Instituto Brasileiro de Geografia e Estatística (2020). Censo demográfico Recife. Rio de Janeiro.

Brasil. Departamento de Informática do SUS (2020). Sistema de Informação de Agravos de Notificação, Ministério da Saúde. Brasília: Ministério da Saúde.

Kanchar, A \& Swaminathan, S. (2019). Tuberculosis Control: WHO Perspective and Guidelines. Indian journal of pediatrics, 86(8), 703-706. https://doi.org/10.1007/s12098-019-02989-2

Macedo, R S \& Bohomol, E (2019). Validation of self-assessment instrument for the Patient Safety Center. Revista brasileira de enfermagem, 72(suppl 1), 259-265. https://doi.org/10.1590/0034-7167-2017-0657

Mashabela, G T; Wet, T J \& Warner, D F (2019). Mycobacterium tuberculosis Metabolism. Microbiology spectrum, 7(4), 10.1128/microbiolspec.GPP3-00672019. https://doi.org/10.1128/microbiolspec.GPP3-0067-2019

Pelissari D M, Bartholomay P, Jacobs M G, Arakaki-Sanchez D, Anjos D S O D, Costa M L D S, Cavalcanti P C D S, Díaz-Quijano F A (2018). Oferta de serviços de atenção básica e detecção da incidência de tuberculose no Brasil. Rev Saúde Pública. 52: 53. doi: 10.11606 / s1518-.

Pasquali L (2010). Instrumento psicológico: Fundamentos e práticas. Porto Alegre: Artmed.

Ribeiro, P L; Cherubim, D O; Padoin, S \& Paula, C C. (2020). Creation and validation of a visual educational technology content for lactation physiology learning. Revista brasileira de enfermagem, 73(6), e20190564. https://doi.org/10.1590/0034-7167-2019-0564.

Simmons, B; Lanuza, D; Fonteyn, M; Hicks, F; \& Holm, K (2003). Raciocínio clínico em enfermeiras experientes. Western Journal of Nursing Research , 25 (6), 701-719.

Sousa, F. das C A, Soares, H V A, Lemos, L E A S, Reis, D M, Silva, W C da, \& Rodrigues, L A de S. (2020). Perfil epidemiológico das doenças de notificação obrigatória negligenciadas no Brasil com análise dos investimentos governamentais na área. Research, Society and Development, 9 (1), e62911610. https://doi.org/10.33448/rsd-v9i1.1610

Tolentino, G S, Bettencourt, A R D C e Fonseca, S M D (2019). Construção e validação de instrumento para consulta de enfermagem em quimioterapia ambulatorial. Revista Brasileira de Enfermagem , 72 , 391-399. 
Research, Society and Development, v. 11, n. 1, e1011124148, 2022

(CC BY 4.0) | ISSN 2525-3409 | DOI: http://dx.doi.org/10.33448/rsd-v11i1.24148

Vieira, M A; Ohara, C V \& Domenico, E B. (2016). The construction and validation of an instrument for the assessment of graduates of undergraduate nursing courses. Revista latino-americana de enfermagem, 24, e2710. https://doi.org/10.1590/1518-8345.0834.2710.

Wisdom, J P; Cavaleri, M A; Onwuegbuzi, A J \& Green C A (2012). Relatórios metodológicos em artigos de pesquisa qualitativa, quantitativa e de métodos mistos em serviços de saúde. Health Serv Res; 47 (2): 721-45. doi: 10.1111 / j.1475-6773.2011.01344. x. 